\title{
Rational Approximations to Generalized Hypergeometric Functions
}

\section{By Jerry L. Fields}

I. Introduction and Summary. In [1], Bellman gave a formal scheme for developing rational approximations to the exponential integral, and the error function. In [2], Luke formally generalized Bellman's scheme to include functions defined by Laplace type integrals, paying particular attention to the generalized hypergeometric functions (see Section II for definition)

$$
\begin{aligned}
&{ }_{p+1} F_{q}\left(\begin{array}{c}
\sigma, \alpha_{p} \\
\rho_{q}
\end{array} \mid \lambda / z\right)=\frac{z^{\sigma}}{\Gamma(\sigma)} \int_{0}^{\infty} e^{-z t} t^{\sigma-1}{ }_{p} F_{q}\left(\begin{array}{c}
\alpha_{p} \\
\rho_{q}
\end{array} \mid \lambda t\right) d t, \\
& q \geqq p+1, \quad \operatorname{Re}(\sigma)>0 .
\end{aligned}
$$

In specific cases, both Bellman and Luke made certain choices of free parameters in their formal schemes and obtained rational approximations which gave excellent agreement with the true values. Except in very special cases, however, neither author proved convergence of the generated rational approximations to the desired limit.

In Theorems I and II, we develop representations for the error of very general types of rational approximations to certain broad classes of generalized hypergeometric functions. As an immediate application of these representations, Corollaries II and V prove that the rational approximations developed by Bellman and Luke actually converge to the desired limit. In Section V, the efficiency of these rational approximations are displayed in two numerical examples.

The representations in Theorems I and II are derived essentially by showing that the error satisfies a certain nonhomogeneous differential equation, and then representing the error in terms of the solutions of the corresponding homogeneous differential equation, using Lagrange's method of variation of parameters. The analysis is related to Lanczos $\tau$-method, see [3]-[5], and resembles in several aspects an analysis of Weber, as given in [6], concerning the Hankel functions $H_{v}^{(i)}(z)$, $i=1,2$.

II. Notation and Basic Development. The generalized hypergeometric function ${ }_{p} F_{q}(z)$, see [7], is defined by the formal expression

$$
{ }_{p} F_{q}(z)={ }_{p} F_{q}\left(\begin{array}{c}
\alpha_{1}, \cdots, \alpha_{p} \\
\rho_{1}, \cdots, \rho_{q}
\end{array} \mid z\right)=\sum_{k=0}^{\infty} \frac{\prod_{j=1}^{p}\left(\alpha_{j}\right)_{k}}{\prod_{j=1}^{q}\left(\rho_{j}\right)_{k}} \cdot \frac{z^{k}}{k !},
$$

where

$$
(\sigma)_{\mu}=\frac{\Gamma(\sigma+\mu)}{\Gamma(\sigma)} .
$$

Received February 18, 1965. This research was sponsored by the Aeronautical Research Laboratories, Office of Aerospace Research, Wright-Patterson Air Force Base, Ohio, under Contract AF 33(657)-8872, Project 7071, "Mathematical Techniques of Aeromechanics," Task 70437, and formed the greater part of the author's doctoral dissertation at Harvard University (June 1964). The author wishes to thank his thesis advisor Professor Joseph L. Walsh and Yudell L. Luke for their many conversations and suggestions. 
We assume that no $\alpha_{j}$ is equal to any $\rho_{j}$, and that no $\rho_{j}$ is a nonpositive integer. For ease in writing, we employ the contracted notation

$$
{ }_{p} F_{q}(z)={ }_{p} F_{q}\left(\begin{array}{c}
\alpha_{p} \\
\rho_{q}
\end{array} \mid z\right)=\sum_{k=0}^{\infty} \frac{\left(\alpha_{p}\right)_{k}}{\left(\rho_{q}\right)_{k}} \cdot{ }_{k !}^{z^{k}} .
$$

Thus $\left(\alpha_{p}\right)_{k}$ is to be interpreted as $\prod_{j=1}^{p}\left(\alpha_{j}\right)_{k}$ and similarly for $\left(\rho_{q}\right)_{k}$. Considered as a power series in $z,{ }_{p} F_{q}(z)$ has a radius of convergence equal to infinity if $p \leqq q$, unity if $p=q+1$, and (in general) zero if $p \geqq q+2$. From (2.2) we see that if some $\alpha_{j}$ is a negative integer $(-n)$,

$$
(-1)^{k} \frac{(-n)_{k}}{k !}=\frac{\Gamma(n+1)}{\Gamma(n-k+1) \Gamma(k+1)}=\left(\begin{array}{l}
n \\
k
\end{array}\right),
$$

and the infinite series (2.1) terminates at $k=n$. If no $\alpha_{j}$ is a negative integer, a meaning can still be given to ${ }_{p} F_{q}(z), p \geqq q+2$, by considering it as the asymptotic expansion as $z \rightarrow 0$, of a certain type of contour integral.

More generally, we define Meijer's $G$-function, see [7], by

$$
G_{p, q}^{m, n}\left(z \mid \begin{array}{c}
\alpha_{1}, \cdots, \alpha_{p} \\
\rho_{1}, \cdots, \rho_{q}
\end{array}\right)=\frac{1}{2 \pi i} \int_{L} \frac{\prod_{j=1}^{m} \Gamma\left(\rho_{j}-s\right) \prod_{j=1}^{n} \Gamma\left(1-\alpha_{j}+s\right)}{\prod_{j=m+1}^{q} \Gamma\left(1-\rho_{j}+s\right) \prod_{j=n+1}^{p} \Gamma\left(\alpha_{j}-s\right)} z^{*} d s
$$

where an empty product is interpreted as $1,0 \leqq m \leqq q, 0 \leqq n \leqq p$, and the parameters are such that no pole of $\Gamma\left(\rho_{j}-s\right), j=1, \cdots, m$ coincides with any pole of $\Gamma\left(1-\alpha_{k}+s\right), k=1, \cdots, n$. Then the contour integral in (2.5) is defined for at least one of the following contours $L_{j}$.

$L_{1}$ runs from $-i \infty$ to $+i \infty$ so that all poles of $\Gamma\left(\rho_{j}-s\right), j=1, \cdots, m$, are to the right, and all the poles of $\Gamma\left(1-\alpha_{k}+s\right), k=1, \cdots, n$, to the left of $L_{1}$. The integral converges if $p+q<2(m+n)$ and $|\arg z|<[m+n-(p+q) / 2] \pi$.

$L_{2}$ is a loop starting and ending at $+\infty$, and encircling all poles of $\Gamma\left(\rho_{3}-s\right)$, $j=1, \cdots, m$, once in the negativedirection, but none of the poles of $\Gamma\left(1-\alpha_{k}+s\right)$, $k=1, \cdots, n$. The integral converges if $q \geqq 1$ and either $p<q$ or $p=q$ and $|z|<1$.

$L_{3}$ is a loop starting and ending at $-\infty$, and encircling all poles of $\Gamma\left(1-\alpha_{k}+s\right)$, $k=1, \cdots, n$, once in the positive direction, but none of the poles of $\Gamma\left(\rho_{j}-s\right)$, $j=1, \cdots, m$. The integral converges if $p \geqq 1$ and either $p>q$ or $p=q$ and $|z|>1$.

If the contour integral in (2.5) is defined for more than one of the $L_{j}$, the resulting contour integrals can be shown to be equal. We let $L$ be a generic notation for any of the $L_{j}$. Near infinity, $L$ can be taken as a straight line parallel to the appropriate axis. Using the same shorthand notation as in (2.3), we rewrite (2.5) as

$$
\begin{aligned}
G_{p, q}^{m, n}\left(z \mid \begin{array}{c}
\alpha_{1}, \cdots, \alpha_{p} \\
\rho_{1}, \cdots, \rho_{q}
\end{array}\right) & =G_{p, q}^{m, n}\left(z \mid \begin{array}{c}
\alpha_{p} \\
\rho_{q}
\end{array}\right) \\
& =\frac{1}{2 \pi i} \int_{L} \frac{\Gamma\left(\rho_{m}-s\right) \Gamma\left(1-\alpha_{n}+s\right)}{\Gamma\left(1-\rho_{q}+s\right) \Gamma\left(\alpha_{p}-s\right)} z^{s} d s .
\end{aligned}
$$

If all the poles of the integrand in (2.6) are simple, it is easy to see from the residue theorem, that 


$$
G_{p, q}^{m, n}\left(z \mid \begin{array}{l}
\alpha_{p} \\
\rho_{q}
\end{array}\right)
$$

can be represented as a sum of well-defined hypergeometric functions. Also, from the integral definition, it follows that

$$
z^{\sigma} G_{p, q}^{m, n}\left(z \mid \begin{array}{l}
\alpha_{p} \\
\rho_{q}
\end{array}\right)=G_{p, q}^{m, n}\left(z \mid \begin{array}{l}
\sigma+\alpha_{p} \\
\sigma+\rho_{q}
\end{array}\right),
$$

and

$$
G_{p, q}^{m, n}\left(z^{-1} \mid \begin{array}{l}
\alpha_{p} \\
\rho_{q}
\end{array}\right)=G_{q, p}^{n, m}\left(z \mid \begin{array}{l}
1-\rho_{q} \\
1-\alpha_{p}
\end{array}\right) .
$$

Consider now for arbitrary $p$ and $q$,

$$
\begin{aligned}
E_{p, q}(z) & =G_{p, q+1}^{1, p}\left(-z \mid \begin{array}{c}
1-\alpha_{p} \\
0,1-\rho_{q}
\end{array}\right) \\
& =\frac{1}{2 \pi i} \int_{L} \frac{\Gamma(-s) \Gamma\left(s+\alpha_{p}\right)}{\Gamma\left(s+\rho_{q}\right)}(-z)^{s} d s
\end{aligned}
$$

under the assumption that no $\alpha_{j}, j=1, \cdots, p$, is a nonpositive integer. If the contour $L$ is moved $n$ units to the right, and account is taken of the simple poles at $k=0,1, \cdots, n-1$, then

$$
\begin{aligned}
E_{p, q}(z)= & \frac{(-2 \pi i)}{(2 \pi i)} \sum_{k=0}^{n-1} \operatorname{Lim}_{s \rightarrow k}(s-k) \frac{\Gamma(-s) \Gamma\left(s+\alpha_{p}\right)}{\Gamma\left(s+\rho_{q}\right)}(-z)^{s} \\
& +\frac{1}{2 \pi i} \int_{L} \frac{\Gamma(-s-n) \Gamma\left(s+n+\alpha_{p}\right)}{\Gamma\left(s+n+\rho_{q}\right)}(-z)^{s+n} d s \\
= & \sum_{k=0}^{n-1} \frac{\Gamma\left(k+\alpha_{p}\right)}{\Gamma\left(k+\rho_{q}\right)} \cdot \frac{z^{k}}{k !} \\
& +\frac{z^{n}}{2 \pi i} \int_{L} \frac{\Gamma(-s) \Gamma(s+1) \Gamma\left(s+n+\alpha_{p}\right)(-z)^{\bullet}}{\Gamma(s+1+n) \Gamma\left(s+n+\rho_{q}\right)} d s \\
= & \frac{\Gamma\left(\alpha_{p}\right)}{\Gamma\left(\rho_{q}\right)} \sum_{k=0}^{n-1} \frac{\left(\alpha_{p}\right)_{k}}{\left(\rho_{q}\right)_{k}} \cdot \frac{z^{k}}{k !} \\
& +z^{n} G_{p+1, q+2}^{1, p+1}\left(-z \mid \begin{array}{c}
0,1-n-\alpha_{p} \\
0,-n, 1-n-\rho_{q}
\end{array}\right),
\end{aligned}
$$

where $\Gamma\left(\alpha_{p}\right)$ stands for $\prod_{j=1}^{p} \Gamma\left(\alpha_{j}\right)$ and a similar remark holds for $\Gamma\left(\rho_{q}\right)$-such notations will be used throughout. If $p<q+1$ or $p=q+1,|\arg (-z)|<\pi$ and $|z|<1$, then the $G$-function on the right of (2.12) approaches zero as $n \rightarrow \infty$. Equation (2.12) then becomes

$$
\begin{aligned}
& E_{p, q}(z)=\frac{\Gamma\left(\alpha_{p}\right)}{\Gamma\left(\rho_{q}\right)}{ }_{p} F_{q}\left(\begin{array}{c}
\alpha_{p} \\
\rho_{q}
\end{array} \mid z\right) \\
& p<q+1 \text { or } p=q+1, \quad|\arg (1-z)|<\pi .
\end{aligned}
$$

Note that $E_{q+1, q}(z)$ analytically extends ${ }_{q+1} F_{q}(z)$ for $|\arg (1-z)|<\pi$. However, if $p \geqq q+2$, then $E_{p, q}(z)$ is still well-defined, and the formal series 


$$
\frac{\Gamma\left(\alpha_{p}\right)}{\Gamma\left(\rho_{q}\right)}{ }_{p} F_{q}\left(\begin{array}{c}
\alpha_{p} \\
\rho_{q}
\end{array} \mid z\right)=\frac{\Gamma\left(\alpha_{p}\right)}{\Gamma\left(\rho_{q}\right)} \sum_{k=0}^{\infty} \frac{\left(\alpha_{p}\right)_{k}}{\left(\rho_{q}\right)_{k}} \cdot \frac{z^{k}}{k !}
$$

serves as its asymptotic expansion uniformly in a proper sector of the $z$-plane, as $z \rightarrow 0$, see [8]. We denote this relationship by

$$
\begin{aligned}
E_{p, q}(z) \sim & \frac{\Gamma\left(\alpha_{p}\right)}{\Gamma\left(\rho_{q}\right)}{ }_{p} F_{q}\left(\begin{array}{c}
\alpha_{p} \\
\rho_{q}
\end{array} \mid z\right), \\
& \beta=p-(q+1) \geqq 1, \quad|\arg (-z)|<(2+\beta) \pi / 2, \quad z \rightarrow 0 .
\end{aligned}
$$

If in (2.11), we replace $n$ by the integer $k+1-a, a=0$ or 1 , multiply the resulting equation by an arbitrary constant $A_{n, k} \gamma^{k}$ and sum from $k=0$ to $n$, we obtain

$$
\begin{gathered}
E_{p, q}(z) \sum_{k=0}^{n} A_{n, k} \gamma^{k}=\sum_{k=0}^{n} A_{n, k} \gamma^{k} P_{k-a+1}(z) \\
+\frac{z^{1-a}}{2 \pi i} \int_{L} \Gamma(-s) \Gamma(s+1)(-z)^{s} \sum_{k=0}^{n} \frac{A_{n, k} \Gamma\left(s+k-a+1+\alpha_{p}\right)}{\Gamma\left(s+k-a+1+\rho_{q+1}\right)}(\gamma z)^{k} d s, \\
P_{k}(z)=\sum_{j=0}^{k-1} \frac{\Gamma\left(j+\alpha_{p}\right)}{\Gamma\left(j+\rho_{q+1}\right)} z^{j}, \quad P_{0}(z)=0, \quad \rho_{q+1}=1 .
\end{gathered}
$$

Now set

$$
\begin{aligned}
& f_{n}{ }^{[r]}(\gamma)=\sum_{k=r}^{n} A_{n, k} \gamma^{k} ; \quad f_{n}(\gamma)=f_{n}{ }^{[0]}(\gamma) \\
& \varphi_{n}(z, \gamma)=\sum_{k=0}^{n} A_{n, k} \gamma^{k} P_{k-a+1}(z) \\
&=\sum_{r=0}^{n-a} \frac{\Gamma\left(r+\alpha_{p}\right)}{\Gamma\left(r+\rho_{q+1}\right)} z^{r} f_{n}^{[r+a]}(\gamma) \\
&=\sum_{k=a}^{n} z^{-k} \sum_{r=k}^{n} \frac{A_{n, r} \Gamma\left(r-k+\alpha_{p}\right)}{\Gamma\left(r-k+\rho_{q+1}\right)}(\gamma z)^{r}, \\
& F_{n}(z, \gamma)= \frac{z^{1-a}}{2 \pi i} \int_{L} \Gamma(-s) \Gamma(s+1)(-z)^{s} \\
& \qquad \cdot \sum_{k=0}^{n} \frac{A_{n, k} \Gamma\left(s+k-a+1+\alpha_{p}\right)}{\Gamma\left(s+k-a+1+\rho_{q+1}\right)}(\gamma z)^{k} d s \\
&=z^{1-a} \sum_{k=0}^{n} A_{n, k}(\gamma z)^{k} G_{p+1, q+2}^{1, p+1}\left(-z \mid \begin{array}{c}
0,-k+a-\alpha_{p} \\
0,-k+a-\rho_{q+1}
\end{array}\right) .
\end{aligned}
$$

Then (2.16) can be written as

$$
E_{p q}(z)=E_{p, q}^{[n]}(z, \gamma)+R_{p, q}^{[n]}(z, \gamma)
$$

where

(2.25) $\quad E_{p, q}^{[n]}(z, \gamma)=\varphi_{n}(z, \gamma) / f_{n}(\gamma) ; \quad R_{p, q}^{[n]}(z, \gamma)=F_{n}(z, \gamma) / f_{n}(\gamma)$.

Thus $E_{p, q}^{[n]}(z, \gamma)$ is a rational approximation to $E_{p, q}(z)$, and $R_{p, q}^{[n]}(z, \gamma)$ is its corresponding error. For future reference we note that

$$
F_{n}(z, 0)=A_{n, 0} E_{p, q}(z), \quad a=1 .
$$


The significance of the parameter $(a)$ is plainly seen, if in (2.21) one successively sets $z=\gamma^{-1}$ and $\gamma=0$. For then $\varphi_{n}(\infty, 0)$ equals zero if $a=1$, and is not equal to zero if $a=0$. We designate these cases as inhomogeneous and homogeneous, respectively. Classically, the homogeneous and inhomogeneous cases correspond to taking the odd or even convergents of certain continued fractions, see [7] and [9]. For some purposes, one case may be more useful than the other.

We shall now show that $E_{p, q}(z)$ satisfies a certain homogeneous differential equation, and that $F_{n}(z, \gamma)$ satisfies a corresponding nonhomogeneous equation. Let

$$
\begin{aligned}
\mathfrak{F C}_{p, q}(D) & =\prod_{j=1}^{q+1}\left(\delta+\rho_{j}-1\right)-z \prod_{j=1}^{p}\left(\delta+\alpha_{j}\right) \\
& =\left(\delta+\rho_{q+1}-1\right)-z\left(\delta+\alpha_{p}\right), \\
\delta & =z D=z \frac{d}{d z}, \quad \rho_{q+1}=1 .
\end{aligned}
$$

The differential operator $\delta$ has the very nice property that for constants $\rho$ and $\sigma$,

$$
(\delta+\sigma) z^{\rho}=z^{\rho}(\rho+\sigma) .
$$

Applying $\mathfrak{K}_{p, q}(D)$ to $F_{n}(z, \gamma)$ as given by (2.22) ( $\gamma$ is independent of $z$ ), we obtain

$$
\begin{aligned}
\mathcal{F C}_{p, q}(D) F_{n}(z, \gamma) & \frac{1}{2 \pi i} \int_{L} \Gamma(-s) \Gamma(1+s) \sum_{k=0}^{n} \frac{A_{n, k}(-1)^{1-a}(-\gamma)^{k} \Gamma\left(s+k-a+1+\alpha_{p}\right)}{\Gamma\left(s+k-a+1+\rho_{q+1}\right)} \\
= & \frac{1}{2 \pi i} \int_{L} \Gamma(-s) \Gamma(1+s) \sum_{k=0}^{n} \frac{A_{n, k}(-1)^{1-a}(-\gamma)^{k} \Gamma\left(s+k-a+1+\alpha_{p}\right)}{\Gamma\left(s+k-a+1+\rho_{q+1}\right)} \\
& \cdot(-z)^{a+k-a+1}\left\{\left(s+k-a+\rho_{q+1}\right)+(-z)\left(s+k-a+1+\alpha_{p}\right)\right\} d s .
\end{aligned}
$$

Then moving the contour of the first integral in (2.30) one unit to the right, and taking account of the residue at $s=0$, we obtain

$$
\begin{aligned}
& \mathcal{F}_{p, q}(D) F_{n}(z, \gamma)=\frac{(-2 \pi i)}{(2 \pi i)} \operatorname{Limit}_{s \rightarrow 0}\{s \Gamma(-s) \Gamma(1+s) \\
& \left.\quad \sum_{k=0}^{n} \frac{A_{n, k} z^{1-a}(z \gamma)^{k} \Gamma\left(s+k-a+1+\alpha_{p}\right)\left(s+k-a+\rho_{q+1}\right)}{\Gamma\left(s+k-a+1+\rho_{q+1}\right)}(-z)^{s}\right\} \\
& +\frac{z^{1-a}}{2 \pi i} \int_{L} \Gamma(-s-1) \Gamma(2+s) \\
& \cdot \sum_{k=0}^{n} \frac{A_{n, k}(\gamma z)^{k} \Gamma\left(s+k-a+2+\alpha_{p}\right)\left(s+k-a+1+\rho_{q+1}\right)}{\Gamma\left(s+k-a+2-\rho_{q+1}\right)}(-z)^{s+1} d s \\
& +\frac{z^{1-a}}{2 \pi i} \int_{L} \Gamma(-s) \Gamma(1+s) \sum_{k=0}^{n} \frac{A_{n, k}(\gamma z)^{k} \Gamma\left(s+k-a+2+\alpha_{p}\right)}{\Gamma\left(s+k-a+1+\rho_{q+1}\right)}(-z)^{s+1} d s .
\end{aligned}
$$


But since

$$
\Gamma(-s-1) \Gamma(2+s)=-\Gamma(-s) \Gamma(1+s),
$$

the two integrals in (2.31) differ by a minus sign. Taking account of this, we arrive at

$$
\begin{aligned}
\mathscr{H C}_{p, q}(D) F_{n}(z, \gamma) & =\gamma^{a} z S_{n-a}(\gamma z), \\
S_{n-a}(w) & =\sum_{k=0}^{n-a} \frac{A_{n, k+a} \Gamma\left(k+1+\alpha_{p}\right)}{\Gamma\left(k+\rho_{q+1}\right)} w^{k} .
\end{aligned}
$$

From equation (2.26), it is then easy to see that

$$
\mathfrak{H}_{p, q}(D) E_{p, Q}(z)=0 \text {. }
$$

Since $f_{n}(\gamma)$ is independent of $z$, the error $R_{p, q}^{[n]}(z, \gamma)=F_{n}(z, \gamma) / f_{n}(\gamma)$ satisfies essentially the same differential equation as $F_{n}(z, \gamma)$, i.e.,

$$
\mathcal{K C}_{p, q}(D) R_{p, q}^{[n]}(z, \gamma)=\gamma^{a} z S_{n-a}(\gamma z) / f_{n}(\gamma) \text {. }
$$

The nature of

$$
\mathfrak{H}_{p, q}(D) y(z)=0
$$

changes drastically as $p \leqq q+1$, or $p \geqq q+2$, and these cases will be treated separately in Sections III and IV, respectively. Our representations of the $R_{p, q}^{[n]}(z, \gamma)$ depend essentially on characterizations of the Green's function for (2.37).

III. The Case $p \leqq q+1$. Keeping the same notations as in Section II, the main result of this section is

THEOREM 1. If, $p$ and $q$ being non-negative integers,

(1) $p \leqq q$ or $p=q+1, z \neq 1$ and $|\arg (1-z)|<\pi$,

(2) $\alpha_{j} \neq$ a negative integer or zero, $j=1, \cdots, p$,

(3) $\operatorname{Re}\left(\rho_{j}\right)>0, j=1, \cdots, q$,

(4) $a=0$ or 1 ,

then there exists a function $t G_{p, q}(z, t) / r_{0}(t)$ which is absolutely integrable with respect to $t$ along any rectifiable path connecting zero and $z$, and with the property that if $A_{n, k} \gamma^{k}, k=0,1, \cdots, n$, are arbitrary constants,

$$
\begin{aligned}
& { }_{p} F_{q}\left(\begin{array}{c}
\alpha_{p} \\
\rho_{q}
\end{array} \mid z\right)=\sum_{k=0}^{n} A_{n, k} \gamma^{k} \sum_{j=0}^{k-a} \frac{\left(\alpha_{p}\right)_{j}}{\left(\rho_{q}\right)_{j}} \cdot \frac{z^{j}}{j !}\left[\sum_{k=0}^{n} A_{n, k} \gamma^{k}\right]^{-1}+\frac{\Gamma\left(\rho_{q}\right)}{\Gamma\left(\alpha_{p}\right)} R_{p, q}^{[n]}(z, \gamma), \\
& \frac{\Gamma\left(\rho_{q}\right)}{\Gamma\left(\alpha_{p}\right)} R_{p, q}^{[n]}(z, \gamma)=a A_{n, 0} \gamma^{a-1}{ }_{p} F_{q}\left(\begin{array}{c}
\alpha_{p} \\
\rho_{q}
\end{array} \mid z\right)\left[\sum_{k=0}^{n} A_{n, k} \gamma^{k}\right]^{-1} \\
& \quad+(-1)^{q} \gamma^{a} \int_{0}^{z} \frac{t G_{p, q}(z, t)}{r_{0}(t)} \sum_{k=0}^{n-a} A_{n, k+a} \frac{\left(\alpha_{p}\right)_{1+k}}{\left(\rho_{q}\right)_{k}} \cdot \frac{(\gamma t)^{k}}{k !} d t\left[\sum_{k=0}^{n} A_{n, k} \gamma^{k}\right]^{-1} .
\end{aligned}
$$

In the notation of Section II, (3.2) is equivalent to

$$
F_{n}(z, \gamma)=a \frac{\Gamma\left(\alpha_{p}\right)}{\Gamma\left(\rho_{q}\right)} A_{n, 0} \gamma_{p}^{a-1} F_{q}\left(\begin{array}{c}
\alpha_{p} \\
\rho_{q}
\end{array} \mid z\right)+(-1)^{q} \gamma^{a} \int_{0}^{z} \frac{t G_{p, q}(z, t)}{r_{0}(t)} S_{n-a}(\gamma t) d t .
$$

It follows from (2.33) that to establish (3.3) we merely need to represent $F_{n}(z, \gamma)$ in terms of the solutions of 


$$
\begin{aligned}
\mathfrak{H}_{p, q}(\mathcal{D}) y(z) & =\left\{\left(\delta-1+\rho_{q+1}\right)-z\left(\delta+\alpha_{p}\right)\right\} y(z) \\
& =\sum_{j=0}^{q+1} r_{j}(z) D^{q+1-j} y(z)=0,
\end{aligned}
$$

using Lagrange's method of variation of parameters. The nature of $\mathfrak{H}_{p, q}(D)$ follows directly from the result, see [10],

$$
\prod_{j=1}^{m}\left(\delta+a_{j}\right)=\sum_{j=0}^{m} \sigma_{m, j} z^{m-j} \mathfrak{D}^{m-j}, \quad \sigma_{m, 0}=1,
$$

where the $a_{j}, \sigma_{m, j}$ are constants. In particular, we see that $z=0$ is a regular singular point of (3.4), and that

$$
\begin{aligned}
r_{0}(z) & =z^{q+1} & & \text { if } p<q+1, \\
& =z^{q+1}(1-z) & & \text { if } p=q+1 .
\end{aligned}
$$

Under the assumption

$$
\rho_{i}-\rho_{j} \neq \text { integer or zero, } \quad i \neq j, \quad i, j=1, \cdots, q+1, \quad \rho_{a+1}=1,
$$

it is easy to show by direct computation that the following functions form a basis of solutions around $z=0$ for (3.4),

$$
y_{j}(z)=z^{1-\rho_{j}}{ }_{1+p} F_{1+q}\left(\begin{array}{c}
1,1+\alpha_{p}-\rho_{j} \\
1+\rho_{q+1}-\rho_{j}
\end{array} \mid z\right), \quad j=1, \cdots, q+1 .
$$

Notice that the hypergeometric functions in (3.8) are actually ${ }_{p} F_{q}$ 's, as one of the denominator parameters is one, which cancels with the first numerator parameter. It follows directly from (3.7) and (3.8) that, if $m$ is a non-negative integer,

$$
\begin{aligned}
& y_{j}^{(m)}(z)=(-1)^{m}\left(\rho_{j}-1\right)_{m}(z)^{1-\rho_{j}-m}{ }_{1+p} F_{1+q}\left(\begin{array}{r}
1,1+\alpha_{p}-\rho_{j} \\
2-\rho_{j}-m, 1+\rho_{q}-\rho_{j}
\end{array} \mid z\right), \\
& j=1, \cdots, q, j=1, \\
& y_{q+1}^{(m)}(z)=\frac{\left(\alpha_{p}\right)_{m}}{\left(\rho_{q}\right)_{m}} F_{q}\left(\begin{array}{l}
m+\alpha_{p} \\
m+\rho_{q}
\end{array} \mid z\right) .
\end{aligned}
$$

Now set

$$
\begin{gathered}
W(t)=\left|\begin{array}{c}
y_{1}{ }^{(0)}(t), \cdots, y_{q+1}^{(0)}(t) \\
\cdots \cdots \cdots \cdots \cdot \cdots \\
y_{1}{ }^{(q)}(t), \cdots, y_{q+1}^{(q)}(t)
\end{array}\right|, \\
G_{p, q}(z, t)=\frac{\left|\begin{array}{c}
y_{1}(z), \cdots, y_{q+1}(z) \\
y_{1}{ }^{(0)}(t), \cdots, y_{q+1}^{(0)}(t) \\
\cdots \cdots \cdots \cdots \\
y_{1}^{(q-1)}(t), \cdots, y_{q+1}^{(q-1)}(t)
\end{array}\right|}{W(t)}=\sum_{j=1}^{q+1} \frac{y_{j}(z) w_{j}(t)}{W(t)} .
\end{gathered}
$$

Making use of the elementary results

$$
\left|\begin{array}{c}
\left(\sigma_{1}\right)_{0}, \cdots,\left(\sigma_{q}\right)_{0} \\
\left(\sigma_{1}\right)_{1}, \cdots,\left(\sigma_{q}\right)_{1} \\
\cdots \cdots \cdots \cdots \cdots, \cdots \\
\left(\sigma_{1}\right)_{q-1}, \cdots,\left(\sigma_{q}\right)_{q-1}
\end{array}\right|=\prod_{1 \leqq j<i \leqq q}\left(\sigma_{i}-\sigma_{j}\right)
$$


and

$$
\frac{1}{\prod_{j=1}^{q+1} \rho_{j}}=\sum_{j=1}^{q+1} \frac{(-1)^{j-1}}{\rho_{j} \prod_{j>k \geq 1}\left(\rho_{j}-\rho_{k}\right) \prod_{q+1 \geq r>j}\left(\rho_{r}-\rho_{j}\right)},
$$

one can show by simple manipulations, that

$$
\begin{aligned}
& \frac{w_{j}(t)}{(-1)^{q} r_{0}(t) W(t)}=\frac{(-1)^{j-1} t^{\rho_{j}-2}\{1+O(t)\}}{\prod_{j>k \geqq 1}\left(\rho_{j}-\rho_{k}\right) \prod_{q+1 \geq r>j}\left(\rho_{r}-\rho_{j}\right)}, \\
& j=1, \cdots, q+1, t \rightarrow 0,
\end{aligned}
$$

and

$$
\int_{0}^{z} \frac{G_{p, q}(z, t) \gamma^{a} t S_{n-a}(\gamma t)}{(-1)^{a} r_{0}(t)} d t=\frac{\Gamma\left(1+\alpha_{p}\right)}{\Gamma\left(1+\rho_{q}\right)} A_{n, a} \gamma^{a} z\{1+O(z)\}, \quad z \rightarrow 0 .
$$

Thus under the hypothesis of Theorem 1 and the conditions (3.7), the function $t G_{p, q}(z, t) / r_{0}(t)$ is absolutely integrable between $t=0$ and $t=z$ (if $p=q+1$, the path connecting 0 and $z$ must avoid the ray from $t=1$, to $t=+\infty)$. Hence, (2.33) implies that for proper connecting constants $C_{j}(\gamma, n)$,

$$
\begin{aligned}
F_{n}(z, \gamma)=\sum_{j=1}^{q+1} C_{j}(\gamma, n) z^{1-\rho_{j}}{ }_{1+p} F_{1+q}\left(\begin{array}{r}
1,1+\alpha_{p}-\rho_{j} \\
1+\rho_{q+1}-\rho_{j}
\end{array} \mid z\right) \\
\quad+\int_{0}^{z} \frac{G_{p, q}(z, t) \gamma^{a} t S_{n-a}(\gamma t)}{(-1)^{a} r_{0}(t)} d t .
\end{aligned}
$$

From $(2.23)$ we see that $F_{n}(z, \gamma)$ is actually analytic at $z=0$ for $p \leqq q+1$, and by comparing coefficients of $z$ as $z \rightarrow 0$, we conclude that

$$
\begin{aligned}
C_{j}(\gamma, n) & =0, \quad j=1, \cdots, q, \\
C_{q+1}(\gamma, n) & =a \frac{\Gamma\left(\alpha_{p}\right)}{\Gamma\left(\rho_{q}\right)} A_{n, 0} \gamma^{a-1} .
\end{aligned}
$$

Equation (3.16) then reduces to (3.3) under the restrictions of (3.7). However, these restrictions can be reduced to hypothesis (3) of Theorem 1 , by noticing that for $t \neq 0, t G_{p, q}(z, t) / r_{0}(t)$ remains well-defined and is absolutely integrable from $t=0$ to $t=z$ when proper limits are taken. We remark that this condition (3) of the hypothesis is not overly stringent, as (2.12) reduces for $p \leqq q+1$ to

$$
{ }_{p} F_{q}\left(\begin{array}{c}
\alpha_{p} \\
\rho_{q}
\end{array} \mid z\right)=\sum_{j=0}^{m-1} \frac{\left(\alpha_{p}\right)_{j}}{\left(\rho_{q}\right)_{j}} \frac{z^{j}}{j !}+\frac{\left(\alpha_{p}\right)_{m} z^{m}}{\left(\rho_{q}\right)_{m}(1)_{m}}{ }_{p+1} F_{q+1}\left(\begin{array}{c}
1, m+\alpha_{p} \\
m+1, m+\rho_{q}
\end{array} \mid z\right)
$$

and Theorem 1 is applicable to the ${ }_{p+1} F_{q+1}$ of (3.18) for $m$ large enough.

Theorem 1 leads immediately to the following results.

Corollary I. If in Theorem 1,

$$
\gamma=r / z, \quad 0<r \leqq 1,
$$

(2) $\operatorname{Lim}_{n \rightarrow \infty} \operatorname{Max}_{0 \leqq t / z \leqq 1}\left|\sum_{k=0}^{n-a} \frac{A_{n, k+a} \Gamma\left(k+1+\alpha_{p}\right)}{\Gamma\left(k+\rho_{q}\right)} \cdot \frac{(r t / z)^{k}}{k !}\right| / \sum_{k=0}^{n} A_{n, k}(r / z)^{k}=0$, 


$$
\operatorname{Lim}_{n \rightarrow \infty} A_{n, 0} / \sum_{k=0}^{n} A_{n, k}(r / z)^{k}=0, \quad \text { if } \quad a=1,
$$

then the rational approximations

$$
\sum_{k=0}^{n} A_{n, k} \gamma^{k} \sum_{j=0}^{k-a} \frac{\left(\alpha_{p}\right)_{j}}{\left(\rho_{q}\right)_{j}} \frac{z^{j}}{j !} / \sum_{k=0}^{n} A_{n, k} \gamma^{k},
$$

converge to

$$
{ }_{p} F_{q}\left(\begin{array}{c|c}
\alpha_{p} \\
\rho_{q}
\end{array} \mid z\right)
$$

as $n \rightarrow \infty$, for each fixed ratio $r / z$; and the convergence is uniform on compact subsets $C$ of the z-plane which exclude $z=0$, if hypotheses (2) and (3) hold uniformly on $C$ (if $p=q+1$, the points of $C$ must satisfy hypothesis (1) of Theorem 1).

Corollary II. If in Theorem 1,

$$
\begin{gathered}
\gamma=r / z, \quad 0<r \leqq 1, \\
A_{n, k}=\frac{(-n)_{k}(n+\lambda)_{k}\left(-a+\rho_{q}\right)_{k}}{(\tau)_{k}\left(1-a+\alpha_{p}\right)_{k}}, \\
\lambda+2 a+1>\tau+a>0, \quad \rho_{j} \neq a, \quad j=1, \cdots, q,
\end{gathered}
$$

then as $n \rightarrow \infty$, the rational approximations (3.19) converge uniformly to

$$
{ }_{p} F_{q}\left(\begin{array}{c|c}
\alpha_{p} \\
\rho_{q}
\end{array} \mid z\right)
$$

on compact subsets $C$ of the z-plane, which exclude $z=0$ (if $p=q+1$, the points of C must satisfy hypothesis (1) of Theorem 1).

To show that the hypothesis of Corollary II actually satisfies the hypothesis of Corollary I, we note that

$$
\begin{aligned}
S_{n-a}(r t / z)= & \sum_{k=0}^{n-a} \frac{A_{n, k+a} \Gamma\left(k+1+\alpha_{p}\right)}{\Gamma\left(k+\rho_{q}\right)} \cdot \frac{(r t / z)^{k}}{k !} \\
= & \frac{\Gamma\left(1-a+\alpha_{p}\right)(-n)_{a}(n+\lambda)_{a}}{\Gamma\left(-a+\rho_{q}\right)(\tau)_{a}} \\
& \cdot{ }_{2} F_{1}\left(\begin{array}{c}
-n+a, n+\lambda+a \mid r t / z) \\
\tau+a
\end{array} \mid r \begin{array}{c} 
\\
\tau+a
\end{array}\right.
\end{aligned}
$$

and that

$$
\begin{aligned}
f_{n}(r / z) & =\sum_{k=0}^{n} A_{n, k}(r / z)^{k} \\
& ={ }_{q+3} F_{p+1}\left(\begin{array}{c}
-n, n+\lambda,-a+\rho_{q}, 1 \\
\tau, 1-a+\alpha_{p}
\end{array} \mid r / z\right) .
\end{aligned}
$$

Thus $S_{n-a}(r t / z)$ is essentially the classical Jacobi polynomial, see [11], which, under the hypothesis of Corollary II, has a uniform algebraic rate of growth with respect to $n$ on the interval $0 \leqq t z^{-1} \leqq 1$, i.e.,

$$
\begin{gathered}
S_{n-a}(r t / z)=O\left(n^{\sigma_{1}}\right), \quad 0 \leqq t z^{-1} \leqq 1, \quad n \rightarrow \infty, \\
\sigma_{1}=\operatorname{Max}\left\{2 a, 2 a+1+\lambda-2 \tau, a-\tau+\frac{1}{2}\right\} .
\end{gathered}
$$


From [12], the behavior of $f_{n}(r / z)$ under the above hypothesis can be deduced to be essentially of exponential growth with respect to $n$. Let $K(z)$ be a generic notation for a nonzero, continuous function of $z$ independent of $n$. Then for $p \leqq q$,

$$
f_{n}(r / z) \sim \frac{\Gamma(2 n+\lambda) \Gamma(n+1) \Gamma\left(n-a+\rho_{q}\right)(r / z)^{n} K(z)}{\Gamma(n+\lambda) \Gamma(n+\tau) \Gamma\left(n+1-a+\alpha_{p}\right)}, \quad n \rightarrow \infty,
$$

and for $p=q+1$,

$$
\begin{aligned}
f_{n}(r / z) & \sim n^{\sigma_{2}} \cosh n \xi K(z), \quad|\arg (-z)|<\pi, \quad n \rightarrow \infty, \\
& \sim n^{\sigma_{2}} \cosh n \eta K(z), \quad\left|\arg \left(\frac{r}{z}-1\right)\right|<\pi, \quad n \rightarrow \infty, \\
\cosh \xi & =1-2 r / z, \quad \cosh \eta=-1+2 r / z, \\
\sigma_{2} & =\sum_{j=1}^{q} \rho_{j}-\sum_{j=1}^{q+1} \alpha_{j}-\tau+a-q+\frac{1}{2},
\end{aligned}
$$

uniformly on compact subsets $C$ of the $z$-plane, which exclude $z=0$ and satisfy the stated conditions of (3.23), (3.24) - the boundary of $C$ is assumed independent of $n$. These results together with (3.22) imply

$$
\operatorname{Lim}_{n \rightarrow \infty} \operatorname{Max}_{0 \leqq t / z \leqq 1}\left|S_{n-a}(r t / z)\right| / f_{n}(r / z)=0,
$$

which is exactly hypothesis (2) of Corollary I. This is sufficient to prove Corollary II. In fact, the same analysis yields the further result:

CoRollary III. If in Corollary II,

(1) $p \leqq q$,

(2) $\bar{R}_{n}(z)$ denotes the error incurred by truncating the Taylor series of

after $n$ terms, then

$$
{ }_{p} F_{q}\left(\begin{array}{c}
\alpha_{p} \\
\rho_{q}
\end{array} \mid z\right)
$$

$$
\begin{aligned}
R_{p, q}^{[n]}(z, r / z) / \bar{R}_{n}(z)= & O\left\{n^{0}(4 r)^{-n}\right\}, \quad r / z \text { fixed, } \quad 0<r \leqq 1, \quad n \rightarrow \infty, \\
\sigma= & p+a(q-p) \\
& +\operatorname{Max}\left\{a, 2 a+\lambda-\tau+\frac{1}{2}, 2 a+\tau-\frac{1}{2}\right\} .
\end{aligned}
$$

This follows directly from the preceding and (3.18) with $m$ replaced by $n$, i.e., if $p \leqq q$,

$$
\begin{aligned}
\bar{R}_{n}(z) & =\frac{\left(\alpha_{p}\right)_{n} z^{n}}{\left(\rho_{q}\right)_{n}(1)_{n}}{ }_{p+1} F_{q+1}\left(\begin{array}{c}
1, n+\alpha_{p} \\
n+1, n+\rho_{q}
\end{array} \mid z\right) \\
& \sim \frac{\Gamma\left(\rho_{q}\right) \Gamma\left(n+\alpha_{p}\right) z^{n}}{\Gamma\left(\alpha_{p}\right) \Gamma\left(n+\rho_{q}\right) \Gamma(n+1)}, \quad z \text { fixed, } n \rightarrow \infty .
\end{aligned}
$$

IV. The Case $p \geqq q+2$. This case is much more complicated than the one treated in Section III, and the results are to a large degree incomplete. However, results are obtained for the important Whittaker function case. These are included in 
Theorem 2. If, $p$ and $q$ being non-negative integers, either

$$
\begin{gathered}
p=q+2 \text { and }|\arg v|<\pi / 2, \text { or } \\
p=q+2, \quad|\arg v| \leqq \pi / 2 \text { and } \operatorname{Re}(\sigma)>-1-q, \text { or } \\
p=q+3, \quad v>0 \text { and } \operatorname{Re}(\sigma)>-1-q / 2, \\
\quad \sigma=\left[\sum_{j=1}^{p} \alpha_{j}-\sum_{j=1}^{q} \rho_{j}+(q-p) / 2\right](p-q-1)^{-1},
\end{gathered}
$$

prevail, and

$$
\begin{aligned}
& v \neq 0, \\
& \alpha_{j} \neq \text { negative integer or zero, } \quad j=1, \cdots, p, \\
& \operatorname{Re}\left(\rho_{j}\right)>0, \quad j=1, \cdots, q, \\
& a=0 \text { or } 1,
\end{aligned}
$$

then there exists a function $G_{p, q}(v, t) t^{-p}$ which is absolutely integrable with respect to $t$ along the ray $\arg t=\arg v$, connecting $v$ and infinity, and with the property that if $A_{n, k} \gamma^{k}, k=0,1, \cdots, n$, are arbitrary constants,

$$
\begin{aligned}
& G_{p, q+1}^{1, p}\left(v^{-1} \mid \begin{array}{c}
1-\alpha_{p} \\
0,1-\rho_{q}
\end{array}\right) \\
& \quad=\sum_{k=0}^{n} A_{n, k} \gamma^{k} \sum_{j=0}^{k-a} \frac{\Gamma\left(j+\frac{\alpha_{p}}{\Gamma}\right)}{\Gamma\left(j+\rho_{q}\right)} \frac{(-v)^{-j}}{j !}\left[\sum_{k=0}^{n} A_{n, k} \gamma^{k}\right]^{-1}+R_{p, q}^{[n]}\left(-v^{-1}, \gamma\right), \\
& R_{p, q}^{[n]}\left(-v^{-1}, \gamma\right)=a A_{n, 0} \gamma^{a-1} G_{p, q+1}^{1, p}\left(v^{-1} \mid \begin{array}{c}
1-\alpha_{p} \\
0,1-\rho_{q}
\end{array}\right)\left[\sum_{k=0}^{n} A_{n, k} \gamma^{k}\right]^{-1} \\
& +\gamma^{a} \int_{v}^{\infty} G_{p, q}(v, t) t^{-p} \sum_{k=0}^{n-a} \frac{A_{n, k+a} \Gamma\left(1+k+\alpha_{p}\right)}{\Gamma\left(k+\rho_{q}\right)} \cdot \frac{(-\gamma / t)^{k}}{k !} d t\left[\sum_{k=0}^{n} A_{n, k} \gamma^{k}\right]^{-1},
\end{aligned}
$$

where

is the Meijer G-function (see [7]),

$$
G_{p, q+1}^{1, p}\left(\begin{array}{l|c}
v^{-1} & \begin{array}{c}
1-\alpha_{p} \\
0,1-\rho_{q}
\end{array}
\end{array}\right)
$$

$$
\begin{aligned}
G_{p, q+1}^{1, p}\left(v^{-1} \mid \begin{array}{c}
1-\alpha_{p} \\
0,1-\rho_{q}
\end{array}\right) & \sim \frac{\Gamma\left(\alpha_{p}\right)}{\Gamma\left(\rho_{q}\right)}{ }_{p} F_{q}\left(\begin{array}{c}
\alpha_{p} \\
\rho_{q}
\end{array} \mid-v^{-1}\right), \\
& |\arg v|<(1+p-q) \pi / 2, \quad v \rightarrow \infty .
\end{aligned}
$$

In the notation of Section II, (4.3) is equivalent to

$$
\begin{aligned}
F_{n}\left(-v^{-1}, \gamma\right)=a \gamma^{a-1} A_{n, 0} G_{p, q+1}^{1, p}\left(\begin{array}{l}
v^{-1} \\
\begin{array}{c}
1-\alpha_{p} \\
0,1-\rho_{q}
\end{array}
\end{array}\right) \\
\quad+\gamma^{a} \int_{v}^{\infty} G_{p, q}(v, t) t^{-p} \sum_{k=0}^{n-a} S_{n-a}(-\gamma / t) d t .
\end{aligned}
$$

To prove (4.5), we find it conveniens to first make the change of variable $z=-v^{-1}$ in (2.33), which then becomes 


$$
\overline{\mathfrak{K}}_{p, q}(D) F_{n}\left(-v^{-1}, \gamma\right)=(-1)^{p+1} \gamma^{a} S_{n-a}(-\gamma / v),
$$

where

$$
\begin{aligned}
\overline{\mathcal{K}}_{p, q}(D) & =\left\{\left(\delta_{1}-\alpha_{p}\right)+(-1)^{\beta} v\left(\delta_{1}+1-\rho_{q+1}\right)\right\} . \\
\delta_{1} & =v D=v \frac{d}{d v}, \quad \beta=p-(q+1) \geqq 1, \quad \rho_{q+1}=1,
\end{aligned}
$$

$$
S_{n-a}(w)=\sum_{k=0}^{n-a} \frac{A_{n, k+a} \Gamma\left(k+1+\alpha_{p}\right)}{\Gamma\left(k+\rho_{q+1}\right)} w^{k}
$$

We then proceed as in Section III to represent $F_{n}\left(-v^{-1}, \gamma\right)$ in terms of the solutions of

$$
\overline{\mathfrak{K}}_{p, q}(D) y(v)=\sum_{j=0}^{p} \bar{r}_{j}(v) D^{p-j} y(v)=0,
$$

using Lagrange's method of variation of parameters. From (3.5) we see that $v=\infty$ is an irregular singular point of (4.9), and that

$$
\bar{r}_{0}(v)=v^{p} \text {. }
$$

Under the assumption

(4.11) $\rho_{i}-\rho_{j} \neq$ integer or zero,

$$
i \neq j ; \quad i, j=1, \cdots, q+1 ; \quad \rho_{q+1}=1,
$$

it is easy to show by direct computation that the following $q+1$ functions are linearly independent solutions of (4.9) around $v=\infty$,

$$
\begin{aligned}
& y_{j}(v)= G_{p, q+1}^{1, p}\left(v^{-1} \mid \begin{array}{c}
1-\alpha_{p} \\
1-\rho_{j}, 0,1-\rho_{1}, \cdots, *, \cdots, 1-\rho_{q}
\end{array}\right), \\
& j=1, \cdots, q+1, \\
&=v^{\rho_{j}-1} G_{p, q+1}^{1, p}\left(v^{-1} \mid \begin{array}{c}
\rho_{j}-\alpha_{p} \\
0, \rho_{j}-1, \rho_{j}-\rho_{1}, \cdots, *, \cdots, \rho_{j}-\rho_{q}
\end{array}\right), \\
& y_{j}(v) \sim v^{\rho_{j}-1} \frac{\Gamma\left(1+\alpha_{p}-\rho_{j}\right)}{\Gamma\left(1+\rho_{q+1}-\rho_{j}\right)}{ }_{1+p} F_{1+q}\left(\begin{array}{c}
\left.1,1+\alpha_{p}-\rho_{j} \mid-v^{-1}\right), \\
1+\rho_{q+1}-\rho_{j}
\end{array}\right), \\
&|\arg v|<(2+\beta)_{\pi / 2}, \quad v \rightarrow \infty .
\end{aligned}
$$

The *'s in (4.12) and (4.13) mean that the factors $1-\rho_{j}$, and $\rho_{j}-\rho_{j}$, respectively, are omitted. Equation (4.13) follows directly from (4.12) by (2.7), and (4.14) follows from (4.13) by (2.15). Then if $m$ is a positive integer, it follows directly from (4.14) that

$$
\begin{aligned}
& y_{j}{ }^{(m)}(v) \sim(-1)^{m} \frac{\Gamma\left(1+\alpha_{p}-\rho_{j}\right)}{\Gamma\left(1+\rho_{q+1}-\rho_{j}\right)}\left(1-\rho_{j}\right)_{m} v^{\rho_{j}-1-m} \\
& \cdot{ }_{2+p} F_{2+q}\left(\begin{array}{c}
1,1+m-\rho_{j}, 1+\alpha_{p}-\rho_{j} \\
1-\rho_{j}, 1+\rho_{q+1}-\rho_{j}
\end{array} \mid-v^{-1}\right), \\
& \quad j=1, \cdots, q,|\arg v|<(2+\beta)_{\pi / 2}, \quad v \rightarrow \infty,
\end{aligned}
$$




$$
\begin{array}{r}
y_{q+1}^{(m)}(v) \sim(-1)^{m+1} \Gamma(m+1) \frac{\left(\alpha_{p}\right)_{1}}{\left(\rho_{q}\right)_{1}} v^{-1-m}{ }_{p+1} F_{q+1}\left(\begin{array}{c}
1+m, 1+\alpha_{p} \\
2,1+\rho_{q}
\end{array} \mid-v^{-1}\right), \\
\quad|\arg v|<(2+\beta) \pi / 2, \quad v \rightarrow \infty
\end{array}
$$

For the remaining $p-(q+1)=\beta$ solutions of (4.9), we follow Meijer, see [13] Choose the integer $\omega$ such that

$$
\begin{aligned}
\left|\arg \left(v e^{i \theta(h)}\right)\right| & <(\beta+\Delta) \pi, \quad h=1, \cdots, \beta, \\
\theta(h) & =(1-2 \omega-2 h) \pi, \\
\Delta & =\frac{1}{2} \quad \text { if } \quad \beta=1, \\
& =1 \quad \text { if } \beta>1 .
\end{aligned}
$$

Then the functions

$$
\begin{aligned}
y_{q+1+h}(v) & =G_{q+1, p}^{p, 0}\left(v e^{i \theta(h)} \mid \begin{array}{c}
\rho_{q+1} \\
\alpha_{p}
\end{array}\right), \quad h=1, \cdots, \beta, \\
& =g_{h}(v),
\end{aligned}
$$

fill out the basis of solutions of (4.9) around $v=\infty$.

Meijer also gives in [13] the Barnes result,

$$
\begin{gathered}
G_{q+1, p}^{p, 0}\left(z \mid \begin{array}{c}
a_{q+1} \\
b_{p}
\end{array}\right) \sim \frac{(2 \pi)^{(\beta-1) / 2}}{\beta^{1 / 2}} \exp \left\{-\beta z^{1 / \beta}\right\} z^{\gamma} \sum_{j=0}^{\infty} K_{j} z^{-j / \beta}, \\
|\arg z|<(\beta+\Delta) \pi, \quad z \rightarrow \infty, \\
\beta=p-(q+1), \tau=\left[2^{-1}(1-\beta)+\sum_{j=1}^{p} b_{j}-\sum_{j=1}^{q+1} a_{j}\right] \cdot \beta^{-1}, K_{0}=1,
\end{gathered}
$$

which permits us to deduce the growth of the $g_{h}(v)$ for $v$ near infinity. Then since

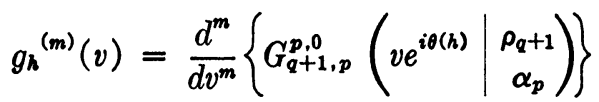

$$
\begin{aligned}
& =G_{q+2, p+1}^{p+1,0}\left(\begin{array}{l|c}
v e^{i \theta(h)} & \begin{array}{c}
-m,-m+1,-m+\rho_{q} \\
0,-m+\alpha_{p}
\end{array}
\end{array}\right)
\end{aligned}
$$

for $m$ a non-negative integer (cancellation occurs among the parameters if $m=0$ ), then it follows from (4.19) that

$$
\begin{gathered}
g_{h}^{(m)}(v) \sim(-v)^{-m}\left[v e^{i \theta(h)}\right]^{m / \beta} \psi_{h}(v) \varphi_{h}(m, v), \\
\left|\arg \left(v e^{i \theta(h)}\right)\right|<(\beta+\Delta) \pi, \quad v \rightarrow \infty, \\
\psi_{h}(v)=\frac{(2 \pi)^{(\beta-1) / 2}}{\beta^{1 / 2}}\left[v e^{i \theta(h)}\right]^{\sigma} \exp \left\{-\beta\left[v e^{i \theta(h)}\right]^{1 / \beta}\right\}, \\
\sigma=\left[\sum_{j=1}^{p} \alpha_{j}-\sum_{j=1}^{q} \rho_{j}-(1+\beta) / 2\right] \beta^{-1}, \\
\varphi_{h}(m, v)=\sum_{j=0}^{\infty} K_{j}(m)\left[v e^{i \theta(h)}\right]^{-j / \beta}, \quad K_{0}(m)=1 .
\end{gathered}
$$


Now set

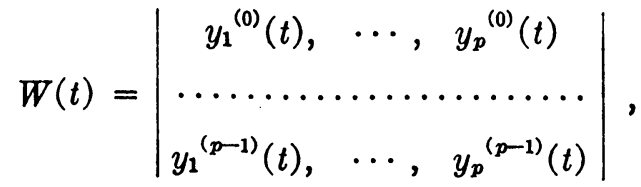

$$
\begin{aligned}
& G_{p, q}(v, t)=\frac{\left|\begin{array}{ccc}
y_{1}(v), & \cdots, & y_{p}(v) \\
y_{1}{ }^{(0)}(t), & \cdots, & y_{p}{ }^{(0)}(t) \\
\cdots \cdots \cdots \cdots \cdots & \ldots \ldots \ldots \cdots \cdots \\
y_{1}{ }^{(p-2)}(t), & \cdots, & y_{p}{ }^{(p-2)}(t)
\end{array}\right|}{W(t)} \\
& =\sum_{j=1}^{p} y_{j}(v) \frac{w_{j}(t)}{W(t)} .
\end{aligned}
$$

It follows then from simple manipulations that

$$
\begin{gathered}
\frac{w_{j}(v)}{\bar{r}_{0}(v) W(v)}=(-1)^{j-1} \frac{\Gamma\left(1+\rho_{q+1}-\rho_{j}\right) v^{-1-\rho_{j}}\left\{1+O\left(v^{-1 / \beta}\right)\right\}}{\Gamma\left(1+\alpha_{p}-\rho_{j}\right) \prod_{1 \leqq r<j}\left(\rho_{j}-\rho_{r}\right) \prod_{j<r \leqq q+1}\left(\rho_{r}-\rho_{j}\right)}, \\
j=1, \cdots, q+1, \quad v \rightarrow \infty, \\
\frac{w_{q+1+h}(v)}{\bar{r}_{0}(v) W(v)}=K_{h} v^{-2-\sigma-q / \beta} \exp \left\{\beta\left[v e^{i \theta(h)}\right]^{1 / \beta}\right\}\left\{1+O\left(v^{-1 / \beta}\right)\right\}, \\
h=1, \ldots, \beta, \quad v \rightarrow \infty
\end{gathered}
$$

where the $K_{h}$ in (4.27) are suitable constants. Equations (4.26) and (4.27) were computed under the restrictions

$$
|\arg v|<(2+\beta) \pi / 2, \quad\left|\arg \left(v e^{i \theta(h)}\right)\right|<(\beta+\Delta) \pi, \quad h=1, \cdots, \beta .
$$

Since $v=\infty$ is an irregular singular point of (4.9), it is not sufficient to consider the ordinary particular solution of (4.9) given by the method of variation of parameters, as was done in Section III. Instead we consider the particular solution

$$
U_{p, q}(v, \gamma)=\sum_{j=1}^{p} y_{j}(v) \int_{\Gamma_{j}(v, \infty)} \frac{w_{j}(t)}{W(t)} \frac{(-1)^{p+1} \gamma^{a} S_{n-a}(-\gamma / t)}{(-1)^{p-1} \bar{r}_{0}(t)} d t,
$$

where $\Gamma_{j}(v, \infty)$ is a path connecting $v$ and $\infty$ in such a manner that the resulting integrands are absolutely integrable along the $\Gamma_{j}(v, \infty)$ and

$$
\operatorname{Lim}_{v \rightarrow \infty} U_{p, q}(v, \gamma)=0
$$

under the restrictions (4.11) and (4.28). For the $\Gamma_{j}(v, \infty), j=1, \cdots, q+1$, it is sufficient to choose the ray, $\arg t=\arg v$. For then, one can show, exactly as in Section III, that

$$
\begin{aligned}
& \sum_{j=1}^{q+1} y_{j}(v) \int_{\Gamma_{j}(v, \infty)} \frac{w_{j}(t) \gamma^{a} S_{n-a}(-\gamma / t)}{W(t) \bar{r}_{0}(t)} d t=\frac{A_{n, a} \Gamma\left(1+\alpha_{p}\right)}{\Gamma\left(1+\rho_{q}\right)} \gamma^{a} v^{-1} \\
& \cdot\left\{1+O\left(v^{-1 / \beta}\right)\right\}, \quad v \rightarrow \infty,
\end{aligned}
$$


under the restrictions (4.11) and (4.28). Then if

$$
\operatorname{Re}(\sigma)>-1-q / \beta,
$$

one possible choice for the $\Gamma_{q+1+h}(v, \infty), h=1, \cdots, \beta$, is as follows. If $\operatorname{Re}\left[v e^{i \theta(h)}\right]^{1 / \beta} \leqq 0$, we choose $\Gamma_{q+1+h}(v, \infty)$ to be the ray, $\arg t=\arg v$. If $\operatorname{Re}\left[v e^{i \theta(h)}\right]^{1 / \beta}>0$, and $\operatorname{Im}\left[v e^{i \theta(h)}\right]^{1 / \beta} \geqq 0$, we choose $\Gamma_{q+1+h}(v, \infty)$ to first proceed along the circle $|v| e^{i \varphi}$, where $\varphi$ increases from zero to $\bar{\varphi}(<\beta \pi / 2), e^{-i \pi / 2}\left[v e^{i(\theta(h)+\bar{\varphi})}\right]^{1 / \beta}>0$, and then along the ray, $\arg t=\arg \bar{\varphi}$. Note that $\left|\exp \left\{\beta\left[v e^{i(\theta(h)+\varphi)}\right]^{1 / \beta}\right\}\right|$ is then a decreasing function of $\varphi$ as $\varphi$ varies between zero and $\bar{\varphi}$. Similarly, if $\operatorname{Re}\left[v e^{i \theta(h)}\right]^{1 / \beta}>0$, and $\operatorname{Im}\left[v e^{i \theta(h)}\right]^{1 / \beta}<0$, we choose $\Gamma_{q+1+h}(v, \infty)$ to first proceed along the circle $|v| e^{i \varphi}$, where $\varphi$ decreases from zero to $\bar{\varphi}(>-\beta \pi / 2), e^{-i \pi / 2}\left[v e^{i(\theta(h)+\overline{\bar{\phi}})}\right]^{1 / \beta}<0$, and then along the ray, $\arg t=\arg \bar{\varphi}$. Making use of (4.27) and the simple fact

$$
\int_{z}^{+\infty} e^{-t} t^{-\alpha} d t=O\left(e^{-z} z^{-\alpha}\right), \quad|\arg z|<\pi, \quad z \rightarrow \infty
$$

it is easy to show that

$$
\sum_{h=1}^{\beta} y_{q+1+h}(v) \int_{\Gamma_{q+1+h}(v, \infty)} \frac{w_{q+1+h}(t) \gamma^{a} S_{n-a}(-\gamma / t)}{W(t) \bar{r}_{0}(t)} d t=O\left(v^{-1-q / \beta}\right), \quad v \rightarrow \infty,
$$

under the restrictions (4.11) and (4.28). Combining (4.31) and (4.34), we see that $U_{p, q}(v, \gamma)$ has the desired property (4.30). Notice that in the cases specified by hypothesis (1) of Theorem 2, the paths $\Gamma_{q+1+h}(v, \infty), h=1, \cdots, \beta$, all reduce to the path, $\arg t=\arg v$.

Then, just as in Section III, since $F_{n}\left(-v^{-1}, \gamma\right)$ and $U_{p, q}(v, \gamma)$ are both solutions of (4.6), we can write for suitable constants $C_{j}(\gamma, n, \omega)$,

$$
F_{n}\left(-v^{-1}, \gamma\right)=\sum_{j=1}^{p} C_{j}(\gamma, n, \omega) y_{j}(v)+U_{p, Q}(v),
$$

where $\omega$ is the integer used to construct the $g_{h}(v)$, see (4.17). Although $F_{n}\left(-v^{-1}, \gamma\right)$ is not analytic at $v=\infty$, it does have a finite limit there, i.e., from (2.23) we can write

$$
\operatorname{Lim}_{v \rightarrow \infty} F_{n}\left(-v^{-1}, \gamma\right)=\frac{a \Gamma\left(\alpha_{p}\right)}{\Gamma\left(\rho_{q}\right)} \gamma^{a-1} A_{n, 0}, \quad|\arg v|<(2+\beta)_{\pi / 2} .
$$

This, together with (4.14), (4.21), (4.30) and (4.35), imply

$$
\begin{aligned}
C_{j}(\gamma, n, \omega) & =0, \quad j=1, \cdots, q, \\
C_{q+1}(\gamma, n, \omega) & =a \gamma^{a-1} A_{n, 0}
\end{aligned}
$$

and

$$
C_{q+1+h}(\gamma, n, \omega)=0
$$

if $\operatorname{Re}\left\{-\beta\left[v e^{i \theta(h)}\right]^{1 / \beta}\right\}>0$ for some particular admissible value of $v$ given by (4.28). For those cases specified by hypothesis (1) of Theorem 2, this determines all the $C_{j}(\gamma, n, \omega), j=1, \cdots, p$. If $\beta \geqq 3$, however, some (not all) of the $C_{q+1+h}(\gamma, n, \omega)$, $h=1, \cdots, \beta$, fail to be defined by (4.38). We have not been able to evaluate the remaining $C_{q+1+h}(\gamma, n, \omega)$ rigorously. For those cases where (4.38) holds for all 
$h, h=1, \cdots, \beta,(4.35)$ reduces to

$$
F_{n}\left(-v^{-1}, \gamma\right)=a \gamma^{a-1} A_{n, 0} G_{p, q+1}^{1, p}\left(\begin{array}{l|l}
v^{-1} & \begin{array}{c}
1-\alpha_{p} \\
0,1-\rho_{q}
\end{array}
\end{array}\right)+U_{p, q}(v, \gamma)
$$

under the restrictions (4.11), which in turn can be weakened to hypothesis (4) of Theorem 2 by taking proper limits. Equation (4.39) is equivalent to (4.5) for those cases specified in Theorem 2 .

Just as Corollaries I and II followed from Theorem 1, the following corollaries follow from Theorem 2.

Corollary IV. If in Theorem 2,

$$
\begin{gathered}
\gamma=r v, \quad 0<r \leqq 1, \\
\operatorname{Lim}_{n \rightarrow \infty} \operatorname{Max}_{0 \leqq v / t \leqq 1}\left|\sum_{k=0}^{n-a} \frac{A_{n, k+a} \Gamma\left(k+1+\alpha_{p}\right)}{\Gamma\left(k+\rho_{q}\right)} \cdot \frac{(-r v / t)^{k}}{k !}\right| / \sum_{k=0}^{n} A_{n, k}(r v)^{k}=0, \\
\operatorname{Lim}_{n \rightarrow \infty} A_{n, 0} / \sum_{k=0}^{n} A_{n, k}(r v)^{k}=0 \text { if } a=1,
\end{gathered}
$$

then the rational approximations

converge to

$$
\sum_{k=0}^{n} A_{n, k} \gamma \sum_{j=0}^{k-a} \frac{\Gamma\left(j+\alpha_{p}\right)}{\Gamma\left(j+\rho_{q}\right)} \cdot \frac{v^{-j}}{j !} / \sum_{k=0}^{n} A_{n, k} \gamma^{k}
$$

$$
G_{p, q+1}^{1, p}\left(v^{-1} \mid \begin{array}{c}
1-\alpha_{p} \\
0,1-\rho_{q}
\end{array}\right) \text { as } n \rightarrow \infty, \text { for each fixed } r v
$$

and the convergence is uniform on compact subsets $C$ of the v-plane which exclude $v=0$, if hypotheses (2) and (3) hold uniformly on $C$.

Corollary V. If in Theorem 2,

$$
\begin{gathered}
\gamma=r v, \quad 0<r \leqq 1, \\
A_{n, k}=\frac{(-n)_{k}(n+\lambda)_{k}\left(-a+\rho_{q}\right)_{k}(-1)^{k}}{(\tau)_{k}\left(1-a+\alpha_{p}\right)_{k}}, \\
\lambda+2 a+1>\tau+a>0, \quad \rho_{j} \neq a, \cdots j=1, \cdots, q,
\end{gathered}
$$

then, as $n \rightarrow \infty$, the rational approximations given by (4.40) converge uniformly to

$$
G_{p, q+1}^{1, p}\left(\begin{array}{l|c}
v^{-1} & \begin{array}{c}
1-\alpha_{p} \\
0,1-\rho_{q}
\end{array}
\end{array}\right)
$$

on compact subsets $C$ of the v-plane which exclude $v=0$.

As before, to show that Corollary V satisfies the hypothesis of Corollary IV, we merely need to note that

$$
\begin{aligned}
& S_{n-a}(-r v / t)=\sum_{k=0}^{n-a} \frac{A_{n, k+a} \Gamma\left(k+1+\alpha_{p}\right)}{\Gamma\left(k+\rho_{q}\right)} \cdot \frac{(-r v / t)^{k}}{k !} \\
&=\frac{\Gamma\left(1-a+\alpha_{p}\right)(-n)_{a}(n+\lambda)_{a}}{\Gamma\left(-a+\rho_{q}\right)(\tau)_{a}} \\
& \cdot{ }_{2} F_{1}\left(\begin{array}{c}
-n+a, n+\lambda+a \mid \\
\tau+a
\end{array} \mid r v / t\right),
\end{aligned}
$$




$$
\begin{aligned}
S_{n-a}(-r v / t) & =O\left(n^{\sigma_{1}}\right), \quad 0 \leqq r v / t \leqq 1, \quad n \rightarrow \infty, \\
\sigma_{1} & =\operatorname{Max}\left\{2 a, 2 a+1+\lambda-2 \tau, a-\tau+\frac{1}{2}\right\}
\end{aligned}
$$

and (see [12]),

$$
\begin{aligned}
f_{n}(r v) & =\sum_{k=0}^{n} A_{n, k}(r v)^{k} \\
& ={ }_{q+3} F_{p+1}\left(\begin{array}{c}
-n, n+\lambda,-a+\rho_{q}, 1 \\
\tau, 1-a+\alpha_{p}
\end{array} \mid-r v\right),
\end{aligned}
$$

$$
\begin{gathered}
f_{n}(r v) \sim K(v) n^{\sigma_{4}} \exp \left\{(2+\beta)\left(r v n^{2}\right)^{(2+\beta)^{-1}}\right\}, \quad|\arg v|<\pi, \quad n \rightarrow \infty, \\
\sigma_{4}=\left[3+\beta+2 a(\beta+1)-2 p-2 \tau+2 C_{1}-2 B_{1}\right](2+\beta)^{-1},
\end{gathered}
$$

where $K(v)$ is a nonzero, continuous function of $v$ independent of $n$, for $v \neq 0$. Equation (4.44) holds uniformly on compact subsets $C$ of the $v$-plane which exclude $v=0$, and satisfy the argument restriction of (4.44)-the boundary of $C$ is assumed independent of $n$. This is sufficient to prove Corollary V.

We note that Corollary V is applicable to the Whittaker function

$$
\begin{aligned}
& W_{\alpha, \mu}(z)=\frac{e^{-z / 2} z^{\kappa}}{\Gamma\left(-\mu-\kappa+\frac{1}{2}\right) \Gamma\left(\mu-\kappa+\frac{1}{2}\right)} \\
& \cdot G_{21}^{12}\left(z^{-1} \mid \begin{array}{c}
-\mu+\kappa+\frac{1}{2}, \mu+\kappa+\frac{1}{2} \\
0
\end{array}\right),
\end{aligned}
$$

and the modified Bessel function

$$
K_{\nu}(z)=\left(\frac{\pi}{2 z}\right)^{1 / 2} W_{0, v}(2 z) .
$$

V. Numerical Examples. Here we give two applications of the preceding results. For the first example, consider the ordinary Bessel function $J_{\nu}(z)$, defined by

$$
{ }_{0} F_{1}\left(1+\nu \mid-z^{2} / 4\right)=\Gamma(1+\nu)(2 / z)^{\nu} J_{\nu}(z) .
$$

If $\operatorname{Re}(1-a+\nu)>0$, Corollary II is applicable, and (3.21), (2.20) reduce to

$$
f_{n}(\gamma)={ }_{4} F_{1}\left(\begin{array}{c|c}
-n, n+\lambda, 1-a+\nu, 1 & \gamma \\
\tau
\end{array}\right),
$$

and

$$
\begin{aligned}
\Gamma(1+\nu) \varphi_{n}\left(-z^{2} / r, \gamma\right) & =\sum_{k=0}^{n-a} \frac{\left(-z^{2} / r\right)^{k}}{(1+\nu)_{k} k !} f_{n}{ }^{[k+a]}(\gamma) \\
& =K_{1} \gamma^{a} \sum_{k=0}^{n-a} \frac{(-n+a)_{k}(n+\lambda+a)_{k}}{(\tau+a)_{k} k !}\left(-\gamma z^{2} / r\right)^{k} \\
& \times{ }_{4} F_{1}\left(\begin{array}{c}
-n+k+a, n+\lambda+k+a, k+1+\nu, 1 \\
\tau+k+a
\end{array}\right), \\
K_{1} & =\frac{(-n)_{a}(n+\lambda)_{a}(1-a+\nu)_{a}}{(\tau)_{a}} .
\end{aligned}
$$


Then setting

$$
J_{\nu}^{[n]}(z, r)=\varphi_{n}\left(-z^{2} / 4,-4 r / z^{2}\right) / f_{n}\left(-4 r / z^{2}\right),
$$

Corollary II implies

$$
(2 / z)^{\nu} J_{\nu}(z)=\operatorname{Lim}_{n \rightarrow \infty} J_{\nu}{ }^{[n]}(z, r), \quad z \text { fixed, } \quad 0<r \leqq 1 .
$$

The following table compares $J_{\nu}{ }^{[n]}(z, r), n=2,3$, 4, with the true values $J_{\nu}(z)$, when $\nu=0, a=0, \lambda=0, \tau=\frac{1}{2}$ and $r=1$ in (5.2) and (5.3).

\begin{tabular}{rrrrr}
\multicolumn{1}{c}{$z$} & \multicolumn{1}{c}{$J_{0}{ }^{[2]}(z, 1)$} & \multicolumn{1}{c}{$J_{0}{ }^{[3]}(z, 1)$} & \multicolumn{1}{c}{$J_{0}{ }^{[4]}(z, 1)$} & \multicolumn{1}{c}{$J_{0}(z)$} \\
\hline 0.5 & 0.9384689 & 0.9384698 & 0.9384698 & 0.9384698 \\
1.0 & 0.7651376 & 0.7651971 & 0.7651977 & 0.7651977 \\
2.0 & 0.2195122 & 0.2237711 & 0.2238899 & 0.2238908 \\
5.0 & -0.6520392 & -0.1936842 & -0.1743481 & -0.1775968 \\
7.0 & -0.3996876 & 0.5794645 & 0.3788970 & 0.3000793 \\
10.0 & 0.0665111 & 0.9984769 & -0.3062838 & -0.2459358
\end{tabular}

The zeros of $J_{0}{ }^{[n]}(z, r)$ also serve as approximations to the zeros of $J_{0}(z)$. The following table compares the zeros of $J_{0}^{[4]}(z, 1)$ with the first eight zeros of $J_{0}(z)$.

\begin{tabular}{lcccccc} 
& First Zeros & Second Zeros & Third Zeros & Fourth Zeros \\
\hline$J_{0}^{[4]}(z, 1)$ & \pm 2.4048169 & \pm 5.4920902 & \pm 9.1653745 & \pm 35.8894094 \\
$J_{0}(z)$ & \pm 2.40482 & 56 & \pm 5.5200781 & \pm 8.6537279 & \pm 11.7915344
\end{tabular}

For our second example, we consider the modified Bessel function $K_{v}(z)$ defined by

$$
\begin{aligned}
K_{\nu}(z) & =e^{-z}\left(\frac{\pi}{2 z}\right)^{1 / 2} E_{\nu}(z) \\
E_{\nu}(z) & =\frac{1}{\Gamma\left(-\nu+\frac{1}{2}\right) \Gamma\left(\nu+\frac{1}{2}\right)} G_{21}^{12}\left(\frac{1}{2 z} \mid \begin{array}{c}
-\nu+\frac{1}{2}, \nu+\frac{1}{2} \\
0
\end{array}\right) \\
& \sim{ }_{2} F_{0}\left(-\nu+\frac{1}{2}, \nu+\frac{1}{2} \mid \frac{-1}{2 z}\right), \quad|\arg z|<3 \pi / 2, z \rightarrow \infty .
\end{aligned}
$$

Thus if $|\arg z| \leqq \pi / 2$, Corollary $\mathrm{V}$ is applicable to $E_{\nu}(z)$, and (4.43), (2.20) reduce to

$$
\begin{gathered}
f_{n}(\gamma)={ }_{3} F_{3}\left(\begin{array}{c}
-n, n+\lambda, 1 \\
\tau,-\nu-a+\frac{3}{2}, \nu-a+\frac{3}{2}
\end{array} \mid-\gamma\right), \\
\varphi_{n}\left(-(2 z)^{-1}, \gamma\right)=\sum_{k=0}^{n-a} \Gamma\left(k-\nu+\frac{1}{2}\right) \Gamma\left(k+\nu+\frac{1}{2}\right) \frac{(-2 z)^{-k}}{k !} f_{n}{ }^{[k+a]}(\gamma) \\
=K_{2}(-\gamma)^{a} \sum_{k=0}^{n-a} \frac{(-n+a)_{k}(n+\lambda+a)_{k}(2 z / \gamma)^{-k}}{(\tau+a)_{k}\left(k-\nu+\frac{1}{2}\right)\left(k+\nu+\frac{1}{2}\right) k !} \\
\quad \times{ }_{3} F_{3}\left(\begin{array}{c}
-n+k+a, n+\lambda+k+a, 1 \\
\tau+k+a,-\nu+k+\frac{3}{2}, \nu+k+\frac{3}{2}
\end{array} \mid-\gamma\right), \\
K_{2}=\Gamma\left(-a+\nu+\frac{3}{2}\right) \Gamma\left(-a-\nu+\frac{3}{2}\right) \frac{(-n)_{a}(n+\lambda)_{a}}{(\tau)_{a}} .
\end{gathered}
$$


Then if we set

$$
E_{\nu}{ }^{[n]}(z, r)=\varphi_{n}\left(-(2 z)^{-1}, 2 r z\right) / f_{n}(2 r z), \quad 0<r \leqq 1,
$$

Corollary V implies

$$
\begin{aligned}
e^{z}[(2 z) / \pi]^{1 / 2} K_{\nu}(z) & =E_{\nu}(z) \\
& =\lim _{n \rightarrow \infty} E_{\nu}^{[n]}(z, r), \quad|\arg z| \leqq \pi / 2, \quad z \text { fixed, } \quad 0<r \leqq 1 .
\end{aligned}
$$

The following table compares $E_{\nu}{ }^{[n]}(z, r), n=2,3,4$, with the true value $E_{\nu}(z)$, when $\nu=0, a=1, \lambda=0, \tau=\frac{1}{2}$ and $r=1$ in (5.10) and (5.11).

\begin{tabular}{rcccc}
\multicolumn{1}{c}{$z$} & $E_{0}{ }^{[2]}(z, 1)$ & $E_{0}{ }^{[3]}(z, 1)$ & $E_{0}{ }^{[4]}(z, 1)$ & $E_{0}(z)$ \\
\hline 0.5 & 0.8679928 & 0.8593268 & 0.8597696 & 0.8598866 \\
1.0 & 0.9148540 & 0.9128349 & 0.9131555 & 0.9131494 \\
2.0 & 0.9495910 & 0.9495496 & 0.9496134 & 0.9496080 \\
5.0 & 0.9772193 & 0.9773567 & 0.9773569 & 0.9773567 \\
7.0 & 0.9833078 & 0.9834019 & 0.9834010 & 0.9834009 \\
10.0 & 0.9880835 & 0.9881400 & 0.9881393 & 0.9881392
\end{tabular}

The table in (5.14) was given previously by Luke [2]. The numbers in (5.14) are especially interesting, if account is taken of the fact that $K_{0}(z)$ has a logarithmic singularity at $z=0$. Numerical computations seem to indicate that the argument restriction, $|\arg z| \leqq \pi / 2$, can be weakened to $|\arg z|<\pi$ at least.

Midwest Research Institute

Kansas City, Missouri

1. R. Bellman, "On approximate expressions for the exponential integral and the error function," J. Math and Phys., v. 30, 1952, pp. 226-231. MR 13, 690.

2. Y. L. LUKE, "On economic representations of transcendental functions," J. Math. and Phys., v. 38, 1960, pp. 279-294. MR 22 *3829.

3. C. LANCzOs, "Trigonometric interpolation of empirical and analytical functions," $J$. Math. and Phys., v. 17, 1938, pp. 123-199.

4. C. Lanczos, Tables of Chebyshev Polynomials $S_{n}(x)$ and $C_{n}(x)$, National Bureau of Standards, AMS9, December, 1952. 823.

5. C. Lanczos, Applied Analysis, Prentice-Hall, Englewood Cliffs, N. J., 1956, MR 18,

6. G. N. WAtson, A Treatise on the Theory of Bessel Functions, 2nd ed., Cambridge Univ. Press, New York and Macmillan, New York, pp. 210-212, MR 6, 64.

7. A. ERDELYI, ET AL., Higher Transcendental Functions, Vol. I, McGraw-Hill, New York, 1953. MR 15, 419 .

8. E. W. BARNES, "The asymptotic expansion of integral functions defined by generalized hypergeometric series," Proc. London Math. Soc. (2), v. 5, 1907, pp. 59-116.

9. Y. L. LuKE, Integrals of Bessel Functions, McGraw-Hill, New York, 1962, pp. 14-17. MR $25 * 5198$.

10. J. L. Fields \& Y. L. LukE, "Asymptotic expansions of a class of hypergeometric polynomials with respect to the order,"J. Math. Anal. Appl., v. 6, 1963, pp. 394-403. MR $26 * 6446$.

11. G. Szeqö, Orthogonal Polynomials, Amer. Math. Soc. Collog. Publ. Vol. 23, Amer. Math. Soc., Providence, R. I., 1939; rev. ed., 1959. MR 1, 14; MR 21 *5029.

12. J. L. Fields \& Y. L. Luke, "Asymptotic expansions of a class of hypergeometric polynomials with respect to the order. II," J. Math. Anal. Appl., v. 7, 1963, pp. 440-451. MR 28 * 256 .

13. C. S. Meijer, "On the G-function. I, II," Nederl. Akad. Wetensch. Proc., v. 49, 1946, p. 234 and pp. $344-346=$ Indag. Math., v. 8, 1946, p. 133 and pp. 213-225. MR 8, 156. 